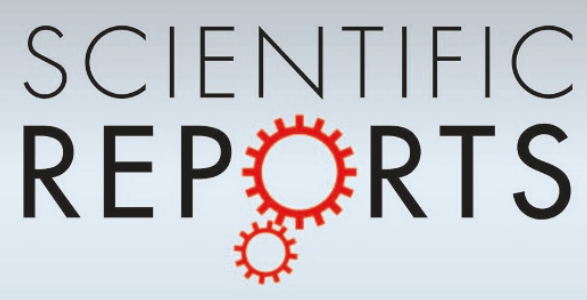

OPEN

SUBJECT AREAS:

TRANSFORMATION

OPTICS

QUANTUM OPTICS

OPTOELECTRONIC DEVICES AND

COMPONENTS

OPTICAL MANIPULATION AND

TWEEZERS

Received

3 October 2014

Accepted

15 December 2014

Published

19 January 2015

Correspondence and requests for materials should be addressed to F.S. (fabio.sciarrino@ uniromal.it)

\section{Arbitrary, direct and deterministic} manipulation of vector beams via electrically-tuned q-plates

\author{
Vincenzo D'Ambrosio' , Flavio Baccari' ${ }^{1}$ Sergei Slussarenko ${ }^{2,3}$, Lorenzo Marrucci ${ }^{2,4}$ \& Fabio Sciarrino'
}

\begin{abstract}
'Dipartimento di Fisica, "Sapienza" Università di Roma, I-00185 Roma, Italy, ${ }^{2}$ Dipartimento di Fisica, Università di Napoli Federico II, Complesso Universitario di Monte S. Angelo, 80126 Napoli, Italy, ${ }^{3}$ Centre for Quantum Dynamics and Centre for Quantum Computation and Communication Technology, Griffith University, Brisbane, Queensland 4111 , Australia, ${ }^{4} \mathrm{CNR}$-SPIN, Complesso Universitario di Monte S. Angelo, 80126 Napoli, Italy.
\end{abstract}

Vectorial vortex light beams, also referred to as spirally polarized beams, are of particular interest since they can be exploited in several applications ranging from quantum communication to spectroscopy and microscopy. In particular, symmetric pairs of vector beams define two-dimensional spaces which are described as "hybrid Poincaré spheres" (HPS). While generation of vortex beams has been demonstrated by various techniques, their manipulation, in particular in order to obtain transformations describing curves entirely contained on a given HPS, is quite challenging, as it requires a simultaneous action on both polarization and orbital angular momentum degrees of freedom. Here, we demonstrate experimentally this kind of manipulation by exploiting electrically-tuned q-plates: an arbitrary transformation on the HPS can be obtained, by controlling two parameters of the q-plate, namely the initial optic axis orientation $\alpha_{0}$ and the uniform birefringent phase retardation $\delta$. Upon varying such parameters, one can determine both the rotation axis and the rotation angle on the HPS, obtaining the desired state manipulation with high fidelity.

$\mathrm{n}$ the past years much interest has been devoted to the study of light beams with a spatially inhomogeneous phase profile like, for instance, eigenstates of the orbital angular momentum (OAM). In general, light can carry both spin and OAM: the first is associated with circular polarizations; the second corresponds to helical wavefronts, as described mathematically by an azimuthal phase factor of the form $\exp (i l \varphi)$, where $\varphi$ is the azimuthal angle in a cylindrical coordinate system around the wave propagation axis $z$ and the topological charge $l$ represents the integer number of crossed helical wavefronts when circling around the axis once. Hence, a circularly polarized photon state with helical wavefront carries a spin of $\sigma \hbar$ (with $\sigma= \pm 1$ ) and an OAM of $l \hbar^{1}$. Typical examples of OAM eigenstates are Laguerre-Gauss or Bessel-Gauss spatial transverse modes ${ }^{2}$. Generation of helical waves requires optical devices that introduce a local phase shift on the beam cross section. Here we consider a particular liquid-crystal-based device, called q-plate (QP) ${ }^{3}$, which has so far been successfully exploited for generation and detection of OAM eigenstates in a variety of applications ${ }^{4}$, ranging from metrology ${ }^{5}$ to fundamental quantum mechanics ${ }^{6}$. Moreover, OAM provides a wide alphabet for qudit encoding for quantum information and computation purposes ${ }^{7}$.

OAM can also be exploited to describe the properties of a class of light beams characterized by a polarization that varies along the azimuthal coordinate $\varphi$ : the so-called vector vortex (VV) beams, also known as spirallypolarized beams. Generation and manipulation of such inhomogeously polarized light beams has been carried out with several techniques, both in the classical ${ }^{8-10}$ and single photon regime ${ }^{11,12}$. An important subfamily of VV beams are those having cylindrical-symmetric polarization patterns, including in particular radial and azimuthal polarized beams ${ }^{13}$. These two last states can be expressed for instance as suitable linear combinations of two helical waves with opposite topological charge $l=+1$ and $l=-1$ and opposite (uniform) circular polarizations. More in general, a linear combination of two OAM eigenstates with eigenvalue $\pm l$ can give rise to an arbitrary VV beam $^{8}$. Symmetric pairs of VV beams in turn define, by linear combination, two-dimensional spaces of nonuniform polarization states formally analogous to the standard Poincare sphere, which have been named in the literature as "hybrid Poincaré spheres" (HPS) $)^{14}$ or "higher-order Poincaré spheres"15. Such inhomogenous polarization states have particular properties that can be exploited to produce strong longitudinal field components ${ }^{16}$ or to obtain a tight focusing ${ }^{17}$. This leads to applications in particle acceleration ${ }^{18}$, spectroscopy ${ }^{19}$, microscopy ${ }^{20}$, optical trapping ${ }^{21}$ and others. Moreover, in a single particle regime, VV beams can be viewed as 
entangled states between spin and orbital angular momentum degrees of freedom ${ }^{22,23}$ and can be expressed via a geometric representation of entangled qubits ${ }^{24-28}$. It has also been recently shown that particular VV states can be exploited for enhanced sensitivity in angular measurements ${ }^{5}$ and rotationally-invariant quantum communication $^{29,30}$. This last result follows from a proposal by Aolita and Walborn ${ }^{31}$ and a proof of principle in classical regime that has been carried out by Souza and co-workers by simulating a physical rotation of reference frame via birefringent waveplates ${ }^{32}$.

In this Letter we introduce and experimentally test a technique, exploiting a particular kind of q-plates with controllable phaseretarding action, that allows a precise and arbitrary manipulation of VV states while remaining within a given HPS space. In other words, we demonstrate arbitrary transformations within these spheres, analogous to the quantum transformations that can be applied on two-state quantum systems, as represented on a Bloch sphere. A pure polarization-to-VV beam conversion process and its reverse one are used for generation and analysis of these states, in order to study the rotation action by means of standard polarization tomography.

\section{Results}

Q-plates general description. Q-plates are liquid-crystal devices that act as interfaces between homogeneously polarized and VV beams via a spin-orbit coupling of the angular momentum of light ${ }^{3,4}$.

A QP is a birefringent plate with a uniform birefringent phase retardation $\delta$ and characterized by an optic axis transverse distribution having a singular pattern with topological charge $q$ and a given initial orientation $\alpha_{0}$. The optic axis pattern is described by the following expression:

$$
\alpha(r, \varphi)=q \varphi+\alpha_{0}
$$

where the QP is supposed lying on the $x y$ plane, described in polar coordinates $(r, \varphi)$ and $\alpha$ denotes the angle formed by the optic axis with the $x$ axis at each point in the plate. Examples of q-plate geometries are shown in Fig. 1. An initially Gaussian circular polarized beam passing through the q-plate is partially or totally converted, depending on the value of $\delta$, into a beam carrying an OAM of $\pm 2 q \hbar$ per photon, with a flipped polarization handedness. More precisely, the QP action on hybrid spin-orbital angular momentum eigenstates can be expressed as follows

$$
Q P\left[\delta, \alpha_{0}\right]|R, l\rangle=\cos \frac{\delta}{2}|R, l\rangle+i e^{-i 2 \alpha_{0}} \sin \frac{\delta}{2}|L, l-2 q\rangle
$$

and
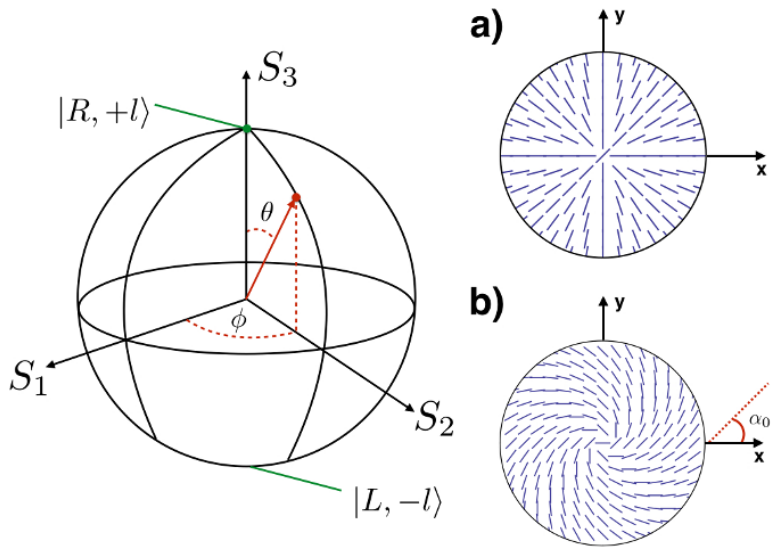

Figure 1 | Left: Hybrid-Poincaré sphere (HPS) representation for a vector vortex beam state of polarization. Right: q-plate geometries for the case $q=1$ and a) $\alpha_{0}=0$, b) $\alpha_{0}=\frac{\pi}{4}$. Blue lines represent local optic axis orientation.

$$
Q P\left[\delta, \alpha_{0}\right]|L, l\rangle=\cos \frac{\delta}{2}|L, l\rangle+i e^{i 2 \alpha_{0}} \sin \frac{\delta}{2}|R, l+2 q\rangle
$$

where $|\pi, l\rangle$ represents a photon state of polarization $\pi$ and carrying an OAM of $i \hbar$. Equation (2) and (3) show that, by acting on the retardation $\delta$, it is possible to vary the coefficients of the modified and unmodified OAM components. Retardation manipulations can be accomplished in several ways, such as by applying pressure, varying the material temperature or, most conveniently, by applying an external electric field ${ }^{33}$. Most of the applications for such optical devices have so far exploited optimal tuning $(\delta=\pi)$ to achieve a complete encoding-decoding process between homogeneous polarization photon states and vector beams $\mathrm{s}^{5,29,34}$. The encoding-decoding system consists in two q-plates with the same topological charge, where the first one converts a given homogeneous polarization state in the corresponding VV and the second one transform the VV back to the homogeneous polarization state. In this way, it is possible to generate and measure VV states with standard optical polarization techniques. The set of the two encoding/decoding q-plates can be considered as a unique device, represented in the $\{|R, 0\rangle,|L, 0\rangle\}$ basis as

$$
E D\left[\alpha_{\text {in }}, \alpha_{\text {out }}\right]=\left(\begin{array}{cc}
e^{2 i\left(\alpha_{\text {out }}-\alpha_{\text {in }}\right)} & 0 \\
0 & e^{-2 i\left(\alpha_{\text {out }}-\alpha_{\text {in }}\right)}
\end{array}\right)
$$

where $\alpha_{\text {in }}$ and $\alpha_{\text {out }}$ denote the $\alpha_{0}$ for the geometry of the first and second q-plate, respectively. Thus, the only visible effect on a linearly polarized beam passing through the device (4) is a polarization rotation of $2\left(\alpha_{\text {out }}-\alpha_{\text {in }}\right)$, rotation which can be easily avoided by rotating one of the q-plates around the $z$ axis until $\alpha_{\text {out }}=\alpha_{i n}$. The only rotationally invariant geometry is the $q=1$ case, where the $\alpha_{0}$ value, corresponding to the $\varphi=0$ optic axis orientation, has to be set in the production process. Hence, for this particular geometry, the condition $\alpha_{\text {out }}=\alpha_{\text {in }}$ is automatically satisfied once the two q-plates are manufactured in the same way.

Manipulating vector beams with q-plates. We now show how a qplate can be exploited to directly manipulate a vector beam state while remaining in a given HPS by acting on the OAM degree of freedom and polarization simultaneously. Suppose that a photon is prepared in a VV state, which can be expressed in the HPS representation $^{15}$ (see Fig. 1) as follows

$$
\cos \frac{\theta}{2}|R,+l\rangle+e^{i \phi} \sin \frac{\theta}{2}|L,-l\rangle,
$$

where $\theta$ and $\phi$ are the polar coordinates of the state in the HPS. Consider then a QP with optical retardation $\delta$ and topological charge $q=l$ acting on the photon state. Through (2) and (3), the transformation applied by the device can be written in the chosen logical basis as:

$$
R\left[\delta, 2 \alpha_{0}\right]=\left(\begin{array}{cc}
\cos \frac{\delta}{2} & i e^{i 2 \alpha_{0}} \sin \frac{\delta}{2} \\
i e^{-i 2 \alpha_{0}} \sin \frac{\delta}{2} & \cos \frac{\delta}{2}
\end{array}\right)
$$

Such unitary transformation represents a logical clockwise state rotation of angle $\delta$ on the HPS sphere, with rotation axis lying on the $x y$ plane (i.e., rotation axis polar angle $\theta_{a x}=\frac{\pi}{2}$ ), with the angle $\alpha_{0}$ that fixes the azimuthal angle of the axis, according to $\phi_{a x}=-2 \alpha_{0}$. This can be shown by considering for instance an incoming $|R,+l\rangle$ state that by (6) is transformed into:

$$
\cos \frac{\delta}{2}|R,+l\rangle+i e^{-2 i \alpha_{0}} \sin \frac{\delta}{2}|L,-l\rangle
$$

The state trajectory thus follows the meridian corresponding to $\phi=$ 
$-2 \alpha_{0}+\pi / 2$, with $\theta$ varying exactly as $\delta$. Moreover, the rotation action described by equation (6) can be exploited to achieve an arbitrary state transformation. Indeed, to reach the state of coordinates $\left\{\theta_{1}, \phi_{1}\right\}$ starting from an initial state $\left\{\theta_{0}, \phi_{0}\right\}$, it is enough to choose the rotation axis (and therefore to set the $\alpha_{0}$ value) so as to have the two states belonging to the same rotation plane. In this way the rotation trajectory connects the two states and the desired transformation can be accomplished, provided the proper $\delta$ value is chosen. (Another possible method consists in combining two QP with proper initial axis orientations, their corresponding rotation matrices chosen to be $R\left[-\theta_{0}, \pi / 2-\phi_{0}\right]$ and $R\left[\theta_{1}, \pi / 2-\phi_{1}\right]$. The first transformation converts the initial $\left\{\theta_{1}, \phi_{1}\right\}$ state in the $|R,+l\rangle$ one, and then the second coincides with (7), $\delta$ and $\alpha_{0}$ values chosen in order to reach the desired final coordinates). As already pointed out, the two parameters $\alpha_{0}$ and $\delta$ can be easily controlled by rotating the q-plate and tuning it respectively, hence an arbitrary transformation is easy to obtain. The only q-plate geometry that does not allow the $\alpha_{0}$ manipulation via rotation is the $q=1$ one, since it is rotationally invariant. In this case $\alpha_{0}$ can be controlled in the manufacturing process as explained in the next section. However, even without a dynamic control of $\alpha_{0}$, it is still always possible to perform an arbitrary transformation on the HPS by using three q-plates (or two, if their order can be exchanged), as explained in the Supplementary Information.

Experiment. Here we describe the first experimental implementation of the rotating device (6) corresponding to an electrically tuned QP with topological charge $q=1$ and $\alpha_{0}=\pi / 4$. This device allows one to perform a logical rotation on the HPS of cylindricallysymmetric beams around the direction $\hat{n}=-\hat{y}$. The experimental setup is shown in Fig. 2 . A laser beam produced by a Ti-Sapphire pulsed laser with wavelength $\lambda=795 \mathrm{~nm}$ is spectrally purified by interference filters with bandwidth $\Delta \lambda=3 \mathrm{~nm}$ and projected onto the spatial mode $T E M_{00}$ by a single mode fiber. The beam is then projected onto the linear polarization state $|H\rangle$ by a polarizing beam splitter (PBS) and then transformed into an arbitrary polarization state via birefringent waveplates. A pure polarization-to- $\mathrm{VV}$ beam conversion process and its reverse one are accomplished by means of two completely tuned $q=1 / 2 \mathrm{q}$-plates, the first one acting as encoder and the second one as decoder. In the first part of the experiment we characterized the electrical response of the $q=1 \mathrm{q}$-plate in order to retrieve the retardation $\delta$ corresponding to a given applied voltage ${ }^{33}$. By removing the two $q=1 / 2$ q-plates from the setting, we measured the intensity of converted $\left(I_{c}\right)$ and unconverted $\left(I_{u}\right)$ components for a circular polarized input beam, as functions of the applied voltage. From $(2,3)$ we know that $I_{u}=I_{0} \cos ^{2} \frac{\delta}{2}$ (where $I_{0}$ stands for the total output intensity), hence we recovered the $\delta$ values corresponding to each applied voltage (see Fig. 2b).

We then verified the logical rotation action of the intermediate qplate for various input states. Our experimental configuration allowed us to visualize the rotation, acting on the VV beam, as a pure polarization one, implying that state preparation as well as projective measurements could be performed by means of ordinary polarization optics. Thus we observed the rotation through polarization tomography of the output state corresponding to different $\delta$ values, ranging from the case of detuned q-plate $(\delta=0)$ to complete tuning $(\delta=\pi)$. By considering the additional phase retardation induced by the $q=1 / 2$ codifying system, it is easy to show that the global threeq-plate device action is represented in the circular polarization basis, apart from global phase factors, by the following matrix:

$$
R_{\mathrm{eff}}=\left(\begin{array}{cc}
e^{2 i\left(\alpha_{\text {in }}-\alpha_{\mathrm{out}}\right)} \cos \frac{\delta}{2} & i e^{2 i\left(\alpha_{\text {in }}+\alpha_{\text {out }}-\alpha_{0}\right)} \sin \frac{\delta}{2} \\
i e^{-2 i\left(\alpha_{\text {in }}+\alpha_{\text {out }}-\alpha_{0}\right)} \sin \frac{\delta}{2} & e^{-2 i\left(\alpha_{\text {in }}-\alpha_{\text {out }}\right)} \cos \frac{\delta}{2}
\end{array}\right)
$$

The linear polarization rotation effect is clearly visible in the $\delta=0$ case, since (8) corresponds exactly to (4). To cancel such effect, we chose $\alpha_{\text {in }}-\alpha_{\text {out }}=0$. This can be done by detuning the $q=1 \mathrm{q}$-plate and rotating the first $q=1 / 2$ with respect to the others (thus varying $\left.\alpha_{i n}\right)$ until an incoming horizontally polarized beam remains un-
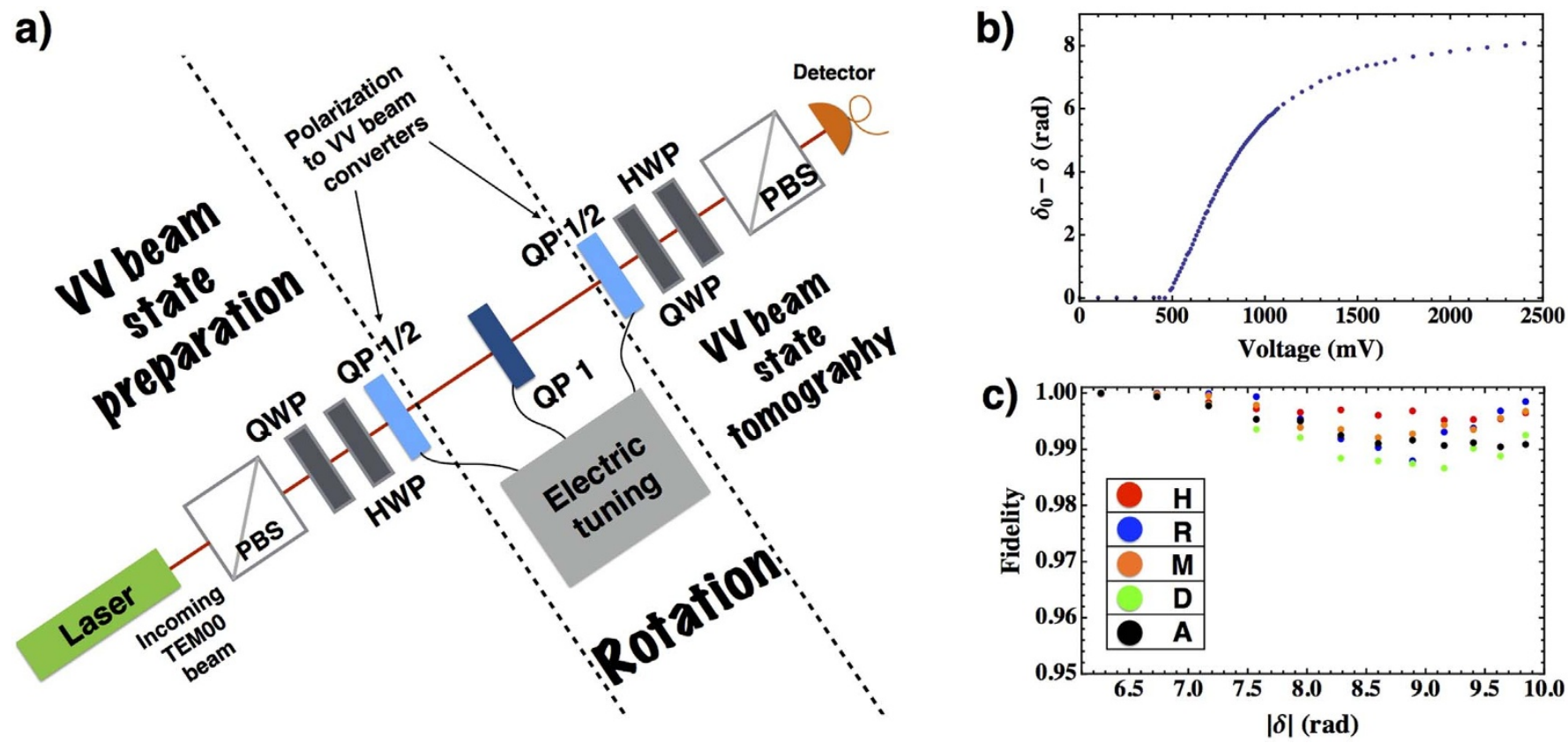

Figure $2 \mid$ (a) Experimental setup. The two q-plates with $q=1 / 2$ act as encoding/decoding system between pure polarization and vectorial vortex beams. Such configuration allows one to accomplish state preparation and detection by means of standard polarization manipulation techniques.

(b) Characterization of the electric response for the $q=1 \mathrm{q}$-plate. The graphic shows the optical retardation $\delta$ corresponding to the voltage applied to the device where $\delta_{0}$ is the optical retardation when no voltage is applied. (c) Fidelity between theoretical and experimental states for progressive $\delta$ values and corresponding to different input states: horizontal $(\mathrm{H})$, circular $(\mathrm{R})$, linear polarization rotated by $22.5^{\circ}$ with respect to $\mathrm{H}(\mathrm{M})$, diagonal $(\mathrm{D})$ and the polarization corresponding to the effective rotation axis (A). Error bars are smaller than experimental points. 

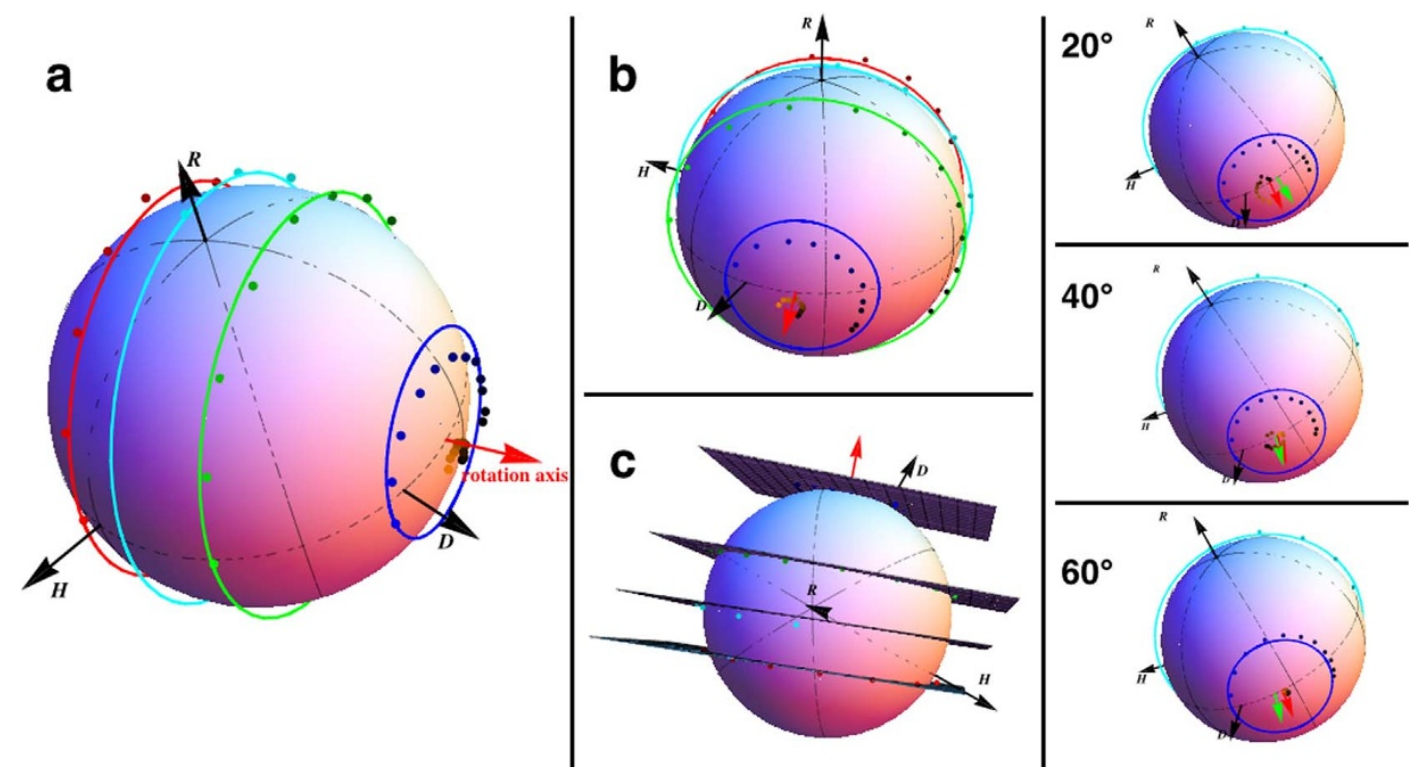

Figure $3 \mid$ Poincaré sphere visualization of the rotation induced by the central $q=1$ q-plate in the HPS. Different colors represent different polarization input states, darkening of the spots corresponding to evolution with $\delta$. Continous lines are the expected theoretical rotation trajectories while the red arrow denotes the rotation axis. a) and b) Rotation viewed from different perspectives. c) Representation of rotation planes for the different input states. Right column: rotation trajectories for three misalignement values between generation and detection stages. Arrows compare the obtained rotation axis (red) with the no misalignement case (green) Error bars are smaller than experimental points.

affected after passing through the system. We point out that by following this method, one ends up with two $q=1 / 2$ sharing the same initial optic axis orientation. Such initial orientation is in general different from that of the $q=1$ one $\left(\alpha_{0} \neq \alpha_{i n}\right)$, this causing a discrepacy between the expected rotation axis, associated with $\alpha_{0}$, and the effective rotation visualized in polarization by means of the codifying system. Indeed, by posing $\alpha_{\text {in }}=\alpha_{\text {out }}=\alpha_{1}$, the (8) coincides with (6) and an effective rotation axis angle $\phi_{a x}=2 \alpha_{0}-4 \alpha_{1}$. Fig. 3 reports our experimental results, showing the rotation effect on the Poincare sphere of polarizations, mapping the equivalent rotation in the HPS.

It can be seen that the rotation planes for each input state are almost parallel, confirming that all the trajectories share the same rotation axis, identified by the red arrow. Since we exploited an $\alpha_{0}=\frac{\pi}{4}$ q-plate, such axis was expected to be exactly the diagonal $|D\rangle=\frac{1}{\sqrt{2}}(|R\rangle+i|L\rangle) \quad$ state. The effective rotation axis is $\frac{1}{\sqrt{2}}[|R\rangle+(-0.33+i 0.94)|L\rangle]$ and the discrepacy between expected and obtained result can be ascribed to an $\alpha_{1}=-0.084$. In order to check the control achievable on the VV state manipulation, we compared the experimental results to the expected trajectories calculated from (8) in correspondence of the same $\delta$ values and with the $\alpha_{1}$ found. The resulting fidelities between experimental and theoretical states are shown in Fig. 2, corresponding to a mean value of $F=$ $0.99485 \pm 0.00006$. We point out that in general, the visualized rotation axis can be modified in order to coincide with the real one by accordingly rotating both $q=1 / 2$ q-plates with respect to the $q=$ 1 one, thus varying $\alpha_{1}$. The desired result (in our case a rotation axis parallel to $|D\rangle$ ) is achieved when $\alpha_{1}=0$.

As a last step, we wanted to confirm that the state manipulation acts on VV beams and not on polarization. Our experimental test was performed on states (5) with $l=1$, which are rotationally invariant, i.e. independent of the relative angle formed by generation and detection setups around the propagation axis. Therefore we mounted the state tomography setup on a rotational stage that can be rotated along the light propagation axis and we repeated measurements for several misalignement angles between the state preparation stage and the state tomography one ${ }^{29}$. Fig. 3 shows that the rotation axis does not depend on the relative rotation between generation and detection stages, thus the rotation action is necessarily acting on the VV beam's state, since the reference frame independence is preserved.

\section{Discussion}

We introduced a technique that allows the arbitrary manipulation of the inhomogeneous state of polarization of vector vortex beams by means of q-plates with controllable parameters such as tuning and initial optic axis orientation. Our experimental implementation considered a single rotating device acting on $\mathrm{VV}$ beams arising form $l=$ \pm 1 OAM eigenstates, corresponding to cylindrically symmetric beams, and we showed that manipulation is indeed achievable with high fidelity independently of the input state considered. Interesting developments could include testing the arbitrary rotation induced by a couple of q-plates with orthogonal rotation axes, as well as exploiting the same manipulation for states of polarization associated with a combination of higher OAM values. Moreover, we point out that alignement-free quantum communication ${ }^{29}$ is related to qubit encoding anologous to (5) with $l=1$. Therefore our work paves the way to manipulation of rotationally-invariant photonic qubits without requiring an encoding-decoding system.

\section{Methods}

Q-plate fabrication methods. The q-plates were manufactured by aligning a nematic liquid crystal (LC) material with the orientation angle given in Eq. (1). The desired planar alignment of the LC was induced using a photoalignment technique ${ }^{35}$. We used $0.1 \%$ wt. solution of Brilliant Yellow (BY) dye in dimethylformamide (DMF) as the aligning surfactant and Indium-Tin-Oxide (ITO) coated glass substrates so to have the possibility of applying an external electrical field to the LC film. For preparing the alignment pattern, a polarized UV laser beam was expanded by a set of lenses, sent through a half-wave plate to set its polarization orientation, and focused with a cylindrical lens, so as to illuminate only a thin azimuthal sector of the LC cell. Both the waveplate and the sample were attached to rotating mounts controlled by a computer, so as to impress sequentially the desired alignment to each azimuthal sector of the LC cell.

1. Allen, L., Beijersbergen, M. W., Spreeuw, R. \& Woerdman, J. Orbital angular momentum of light and the transformation of Laguerre-Gaussian laser modes. Phys. Rev. A 45, 8185 (1992).

2. Siegman, A. E. Lasers (University Science Books. Mill Valley, 1986). 
3. Marrucci, L., Manzo, C. \& Paparo, D. Optical spin-to-orbital angular momentum conversion in inhomogeneous anisotropic media. Phys. Rev. Lett. 96, 163905 (2006).

4. Marrucci, L. et al. Spin-to-orbital conversion of the angular momentum of light and its classical and quantum applications. Journal of Optics 13, 064001 (2011).

5. D'Ambrosio, V. et al. Photonic polarization gears for ultra-sensitive angular measurements. Nat Commun 4, 2432 (2013).

6. D'Ambrosio, V. et al. Experimental implementation of a kochen-specker set of quantum tests. Physical Review X 3, 011012 (2013).

7. D'Ambrosio, V. et al. Test of mutually unbiased bases for six-dimensional photonic quantum systems. Scientific reports 3, 2726 (2013).

8. Maurer, C., Jesacher, A., Fürhapter, S., Bernet, S. \& Ritsch-Marte, M. Tailoring of arbitrary optical vector beams. New Journal of Physics 9, 78 (2007).

9. Krishna Inavalli, V. V. G. \& Viswanathan, N. K. Switchable vector vortex beam generation using an optical fiber. Optics Communications 283, 861-864 (2010).

10. Galvez, E. J., Khadka, S., Schubert, W. H. \& Nomoto, S. Poincare beam patterns produced by nonseparable superpositions of laguerre-gauss and polarization modes of light. Appl. Opt. 51, 2925-2934 (2012).

11. Barreiro, J. T., Wei, T.-C. \& Kwiat, P. G. Remote preparation of single-photon "hybrid" entangled and vector-polarization states. Phys. Rev. Lett. 105, 030407 (2010)

12. Fickler, R., Lapkiewicz, R., Ramelow, S. \& Zeilinger, A. Quantum entanglement of complex photon polarization patterns in vector beams. Phys. Rev. A 89, 060301 (2014).

13. Zhan, Q. Cylindrical vector beams: from mathematical concepts to applications. Advances in Optics and Photonics 1, 1-57 (2009).

14. Holleczek, A., Aiello, A., Gabriel, C., Marquardt, C. \& Leuchs, G. Classical and quantum properties of cylindrically polarized states of light. Opt. Express 19, 9714-9736 (2011)

15. Milione, G., Sztul, H., Nolan, D. \& Alfano, R. Higher-order poincaré sphere, stokes parameters, and the angular momentum of light. Phys. Rev. Lett. 107, 053601 (2011)

16. Novotny, L., Beversluis, M., Youngworth, K. \& Brown, T. Longitudinal field modes probed by single molecules. Phys. Rev. Lett. 86, 5251 (2001).

17. Quabis, S., Dorn, R., Eberler, M., Glöckl, O. \& Leuchs, G. Focusing light to a tighter spot. Optics Communications 179, 1-7 (2000)

18. Kimura, W. et al. Laser acceleration of relativistic electrons using the inverse cherenkov effect. Phys. Rev. Lett. 74, 546 (1995).

19. Dorn, R., Quabis, S. \& Leuchs, G. Sharper focus for a radially polarized light beam. Phys. Rev. Lett. 91, 233901 (2003).

20. Abouraddy, A. F. \& Toussaint Jr, K. C. Three-dimensional polarization control in microscopy. Phys. Rev. Lett. 96, 153901 (2006).

21. Roxworthy, B. J. \& Toussaint Jr, K. C. Optical trapping with $\pi$-phase cylindrical vector beams. New Journal of Physics 12, 073012 (2010).

22. Chen, L. \& She, W. Single-photon spin-orbit entanglement violating a bell-like inequality. J. Opt. Soc. Am. B 27, A7-A10 (2010).

23. Borges, C. V. S., Hor-Meyll, M., Huguenin, J. A. O. \& Khoury, A. Z. Bell-like inequality for the spin-orbit separability of a laser beam. Phys. Rev. A 82, 033833 (2010).

24. Milman, P. \& Mosseri, R. Topological phase for entangled two-qubit states. Phys. Rev. Lett. 90, 230403 (2003).

25. Milman, P. Phase dynamics of entangled qubits. Phys. Rev. A 73, 062118 (2006).
26. LiMing, W., Tang, Z. L. \& Liao, C. J. Representation of the SO(3) group by a maximally entangled state. Phys. Rev. A 69, 064301 (2004).

27. Souza, C. E. R., Huguenin, J. A. O., Milman, P. \& Khoury, A. Z. Topological phase for spin-orbit transformations on a laser beam. Phys. Rev. Lett. 99, 160401 (2007).

28. Souza, C. E. R., Huguenin, J. A. O. \& Khoury, A. Z. Topological phase structure of vector vortex beams. J. Opt. Soc. Am. A 31, 1007-1012 (2014).

29. D'Ambrosio, V. et al. Complete experimental toolbox for alignment-free quantum communication. Nat Commun 3, 961 (2012).

30. Vallone, G. et al. Free-space quantum key distribution by rotation-invariant twisted photons. Phys. Rev. Lett. 113, 060503 (2014).

31. Aolita, L. \& Walborn, S. P. Quantum communication without alignment using multiple-qubit single-photon states. Phys. Rev. Lett. 98, 100501 (2007)

32. Souza, C. E. R. et al. Quantum key distribution without a shared reference frame. Phys. Rev. A 77, 032345 (2008).

33. Piccirillo, B., D’Ambrosio, V., Slussarenko, S., Marrucci, L. \& Santamato, E. Photon spin-to-orbital angular momentum conversion via an electrically tunable q-plate. Applied Physics Letters 97, 241104-241104-3 (2010).

34. Nagali, E. et al. Quantum information transfer from spin to orbital angular momentum of photons. Phys. Rev. Lett. 103, 013601 (2009).

35. Slussarenko, S. et al. Tunable liquid crystal q-plates with arbitrary topological charge. Opt. Express 19, 4085-4090 (2011).

\section{Acknowledgments}

This work was supported by FIRB Futuro in Ricerca-HYTEQ, PRIN (Programmi di ricerca di rilevane interesse nazionale) project AQUASIM, European project QWAD (Quantum Waveguide Application and Devices): www.qwad-project.eu and ERC-Starting Grant 3D-QUEST (3D-Quantum Integrated Optical Simulation; Grant Agreement No. 307783): www.3dquest.eu.

\section{Author contributions}

V.D. and F.S. conceived the concept, S.S. and L.M. fabricated the device, V.D., F.B. and F.S. carried out the experiment and analyzed the data, V.D., F.B., L.M. and F.S. wrote the paper.

\section{Additional information}

Supplementary information accompanies this paper at http://www.nature.com/ scientificreports

Competing financial interests: The authors declare no competing financial interests. How to cite this article: D’Ambrosio, V., Baccari, F., Slussarenko, S., Marrucci, L. \& Sciarrino, F. Arbitrary, direct and deterministic manipulation of vector beams via electrically-tuned q-plates. Sci. Rep. 5, 7840; DOI:10.1038/srep07840 (2015).

This work is licensed under a Creative Commons Attribution-NonCommercialNoDerivs 4.0 International License. The images or other third party material in this article are included in the article's Creative Commons license, unless indicated otherwise in the credit line; if the material is not included under the Creative Commons license, users will need to obtain permission from the license holder in order to reproduce the material. To view a copy of this license, visit http:// creativecommons.org/licenses/by-nc-nd/4.0/ 